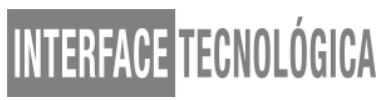

\title{
USO DE TECNOLOGIA NA RASTREABILIDADE DO REBANHO DE CORTE
}

\author{
USE OF TECHNOLOGY IN HERD TRACEABILITY
}

\author{
Igor Augusto Trigo - igor_trigo@ hotmail.com \\ Marcela Midori Yada - marcelayada@gmail.com \\ Ludmila da Silva Lourençano - ludmilalourencano@live.com \\ Yasmin Khatib de Lima - yasmin.khatib@hotmail.com
}

Faculdade de Tecnologia de Taquaritinga (FATEC) - SP - Brasil

DOI: 10.31510/infa.v15i2.464

\begin{abstract}
RESUMO
O presente artigo tem como objetivo apresentar um projeto que propõe uma solução para os produtores de gado de corte, de pequeno ou médio porte, através do uso de tecnologia, para que possam ter acesso a todas as informações necessárias de seu rebanho, desde a quantidade de animais, pesos e vacinas, até o tamanho da área de confinamento, através de um aplicativo, o qual será disponibilizado em todas as plataformas digitais e poderá ser acessado em uma ou mais telas, seja em computadores ou em dispositivos portáteis. Para isso, foi necessário realizar um estudo da área em que seria implantado o sistema e, logo após, com o auxílio de um drone, disponibilizando as informações no aplicativo. Com isso, o produtor poderá economizar tempo e possuir um maior controle sobre seu rebanho, podendo acessar as informações mesmo não estando próximo ao gado. Portanto, além de incluir o uso de tecnologia no dia a dia do produtor, permitirá agilizar os trabalhos a serem realizados no confinamento, permitindo emitir um relatório do lote para as indústrias de abate, garantindo assim, a qualidade do rebanho de corte.
\end{abstract}

Palavras-chave: Bovino de Corte. Plataformas Digitais. Aplicativos.

\begin{abstract}
This paper aims at presenting a project which proposes a solution for small and medium-sized cattle producers based on the use of technology, so that they can access all the needed information about their herd, from the number of animals, their weights and vaccines, to the size of the confinement area, by means of an application, which is offered on all digital platforms and can be accessed on one screen or more, either on computers or on portable devices. For such purpose, we carried out a study of the area in which the system would be implanted and then, with the aid of a drone, we made the information available in the app. Hence, the producer can have greater time and greater control over their herd, being able to access information even when distant. The app then brings to the producer the use of technology in a day-to-day basis, allowing greater agility in the work carried out in the containment, also offering to the slaughter industries a report on the herd lot, thus ensuring the quality of the cattle.
\end{abstract}


Keywords: Beef Cattle. Digital Platforms. Applications.

\section{INTRODUÇÃO}

A criação do gado de corte no Brasil passou por diversas transformações significativas nos últimos dez anos. Isso se deve à ampliação das fronteiras agrícolas no centro-oeste e norte do país, que contribuiu para o crescimento acentuado do efetivo bovino (BARCELLOS et. al., 2004)

No histórico produtivo ocorreu o surgimento e consolidação de novas tecnologias de produção, bem como a suplementação estratégica, o semi-confinamento, uso das misturas múltiplas, cruzamentos, novas variedades, entre outros, o que permitiu encurtar o ciclo de produção (BARCELLOS et. al., 2004).

Além desses fatores, houve a incorporação da gestão tecnológica integrada aos custos e margens econômicas, o que possibilitou a pecuária de corte ser um dos protagonistas do agronegócio brasileiro (BARCELLOS et. al., 2004).

O sistema atual de produção é conhecido na literatura como "dentro da porteira", e caracteriza tudo o que está relacionado a entrada de insumos, ocupação do solo, tecnologia de processos e resultado econômico, ou seja, nada mais é do que uma segmentação da cadeia produtiva, e neste caso, trata-se da pecuária de corte (BARCELLOS et. al., 2004).

O sistema de produção da pecuária de corte estratifica-se nas seguintes etapas: > cria; > recria; > ciclo completo; > recria; > recria - engorda; > engorda. De forma geral, essas etapas citadas passaram a receber fortes investimentos, com o objetivo de intensificar os sistemas, reduzir custos fixos, maximizar a mão-de-obra e consequentemente, melhorar os indicadores de produtividade (BARCELLOS et. al., 2004).

Quanto ao consumidor final, passaram a exigir algumas características ou requisitos de qualidade referentes a carne bovina. Dessa forma, é imprescindível conhecer o mercado em que atuam e o perfil do consumidor que o compõe, sendo uma estratégia de sobrevivência em um mercado competitivo, cujo objetivo é atender as necessidades do consumidor, e consequentemente, o sucesso da empresa (BARCELLOS et. al., 2004; MOTA, 2011).

A respeito da qualidade e inocuidade, a implantação do sistema de rastreamento ocorreu devido a exigência dos países importadores da Comunidade Europeia, onde novas regras de rotulagem entraram em vigor no ano de 2000 e, requerem identificação precisa da 


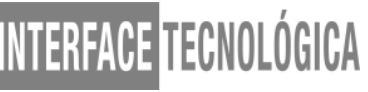

procedência dos produtos de origem animal. Tais medidas buscam restaurar a confiança dos consumidores após a difusão da Encefalopatia Espongiforme Bovina (EBB), comumente conhecida por "doença da vaca louca" (MOTA, 2011).

Mediante isso, para se adequar a essas medidas, o Brasil passou a realizar a rastreabilidade nos seus rebanhos. Dessa forma, foi criada a Instrução Normativa - $\mathrm{IN}^{\circ} 01$, de 09 de janeiro de 2002, publicada no Diário Oficial da União - DOU de 10 de janeiro de 2002, que caracterizam a origem, o estado sanitário, a produção, produtividade da pecuária nacional e segurança dos alimentos provenientes (MOTA, 2011).

O objetivo do trabalho é apresentar um projeto de criação e implantação de um aplicativo de fácil acesso que faz a rastreabilidade de bovinos para produtores de pequeno e/ou médio porte.

\section{FUNDAMENTAÇÃO TEÓRICA}

\subsection{Histórico Produtivo}

O custo de produção da carne brasileira é $60 \%$ mais baixo que o australiano e $50 \%$ mais baixo que o americano, isso torna a carne brasileira muito competitiva no mercado mundial, onde tem ocupado lugar de destaque no cenário mundial da carne. Entretanto, tal competitividade tem exigido ajustes e novos posicionamentos dos agentes da cadeia da carne bovina e do governo, já que o preço final se mostra insuficiente para a consolidação de mercado (MOTA, 2011).

O Brasil possui muitas vantagens naturais e forte tradição exportadora de alimentos junto com o grande mercado interno inserido com o crescente mercado externo representam uma extraordinária oportunidade de consolidação (MOTA, 2011).

Por outro lado, a globalização do mercado cada vez mais disputado, os consumidores aumentam cada vez mais suas exigências quanto a qualidade e a crescente preocupação com a saúde e ecologia junto as mudanças de estilos de vida e o baixo poder aquisitivo do consumidor representam ameaças para o setor desarticulado nas bases da produção, indústria e varejo, como é a cadeia de bovinos hoje (MOTA, 2011).

Sem uma quantidade suficiente de consumidores satisfeitos, não há negócio sustentável nem ganhos satisfatórios. Sendo os consumidores a razão de qualquer estratégia 


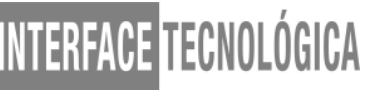

de produção pecuária, deve ser direcionado pelas exigências básicas de mercado exigidas por eles, como: baixo custo, qualidade e ofertas consideráveis de produto final (MOTA, 2011).

O foco no consumidor ganhou grande importância no processo de globalização da economia pelas empresas inseridas nos mercados competitivos e as garantias de qualidade intrínseca aos produtos ou de serviços ou de segurança sanitária são cada vez mais exigidas pelos consumidores e, consequentemente, buscada pelos integrantes das cadeias produtivas (MOTA, 2011).

No segmento da comercialização, é crescente a importância dos supermercados como canal de vendas, porém, como este setor se moderniza rapidamente, seguindo novas exigências e formando novos hábitos de consumo, as relações entre os frigoríficos e supermercados tendem a seguir o padrão da indústria de alimentos em geral, exigindo novas formas de apresentação, qualificação e garantia da carne ofertada (MOTA, 2011).

\subsection{Rastreabilidade}

A rastreabilidade é um sistema de controle que permite a identificação individual e/ou lote do produto, desde seus componentes até o produto final. Com isso, o consumidor tem a garantia de um produto saudável e seguro (MARTINS e LOPES, 2001).

A rastreabilidade tem como principal foco controlar e garantir, individualmente, a qualidade do animal ao longo de sua vida, possibilitando diversas análises a respeito da cadeia produtiva do animal, desde sua origem, até sua alimentação e manejo de vacinação. Com isso, o produtor pode garantir que o animal possua uma qualidade adequada para o consumo humano, seguindo todas as normas necessárias legais (SERVIÇO BRASILEIRO DE CERTIFICAÇÕES, 2018).

A implantação do sistema de rastreamento ocorreu devido a exigência dos países importadores da Europa, onde novas regras de rotulagem entraram em vigor no ano de 2000 e necessitavam identificação precisa da procedência dos produtos que continham carne bovina em sua composição. Tais medidas buscam restaurar a confiança dos consumidores após a difusão da Encefalopatia Espongiforme Bovina - EBB - comumente conhecida por "doença da vaca louca" (MARTINS e LOPES, 2001).

Diante desse cenário, o Brasil buscou se adequar a tais normas e implantou o processo de rastreabilidade e certificação do rebanho, como uma forma de garantia da qualidade e inocuidade (MARTINS e LOPES, 2001). 


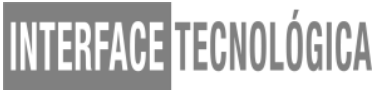

Dessa forma, a utilização da visão sistêmica na cadeia produtiva da carne bovina possibilitou a incorporação de novas tecnologias no sistema produtivo, com destaque para o uso da tecnologia da informação na gestão do empreendimento. A partir da identificação eletrônica e rastreabilidade, permitem que o sistema de dados da propriedade seja alimentado com dados confiáveis, cujos dados são indispensáveis para o planejamento das atividade e melhor coordenação entre os elos da cadeia (MARTINS e LOPES, 2001; LUCHIARI FILHO, 2006).

Assim, na criação de bovinos de corte, a rastreabilidade age desde o nascimento até o abate, passando por todas as fases: produção, industrialização, transporte, distribuição e comercialização, proporcionando uma correlação entre o produto final e a matéria-prima que lhe deu origem (MARTINS e LOPES, 2001).

Com o passar dos anos, os sistemas de rastreabilidade sofreram notável desenvolvimento. Por isso, é imprescindível harmonizar sistemas comprovadamente capazes de melhorar a coerência entre os países implicados nas transações comerciais, afim de garantir melhorias e facilitar os intercâmbios internacionais de animais e produtos de origem animal (MARTINS e LOPES, 2001).

Mediante isso, os sistemas de rastreabilidade podem ser subdivididos em quatro categorias, segundo sua aplicação, bem como, no país de origem, no varejista, na indústria ou na identificação do animal desde a fazenda até a venda do produto final (MARTINS e LOPES, 2001).

A despeito do uso de tecnologia, que aumentam a rapidez e exatidão dos processos de obtenção e manipulação dos dados, deve existir uma independência entre os sistemas ao mesmo passo em que eles devem ser compatíveis (MARTINS e LOPES, 2001).

A rastreabilidade é de suma importância para possibilitar ao Ministério da Agricultura, Pecuária e Abastecimento (MAPA), ter informações mais detalhadas sobre o rebanho nacional, e principalmente, assegurar aos consumidores o estado sanitário e nutricional dos animais, que devem estar de acordo com os padrões pré-estabelecidos de qualidade (MARTINS e LOPES, 2001).

Também atua em evidenciar a eficiência do sistema produtivo, propondo controle da vida do animal, facilitando assim, a localização de focos de doenças e sua possível solução (JUSTINO, 2002). 


\subsection{Mercado da carne bovina}

A expectativa para o mercado de carne bovina está otimista para o ano de 2018, com um crescimento de $5 \%$ em relação aos anos anteriores (Figura 1). Em relação a produção global, deverá ter um aumento de $2 \%$, referente a 63,0 milhões de toneladas. Já em relação as exportações globais, é previsto um aumento de 5\%, representando 10,5 milhões de toneladas.

Figura 1 - Exportação em milhões de toneladas da carne bovina em 2018.

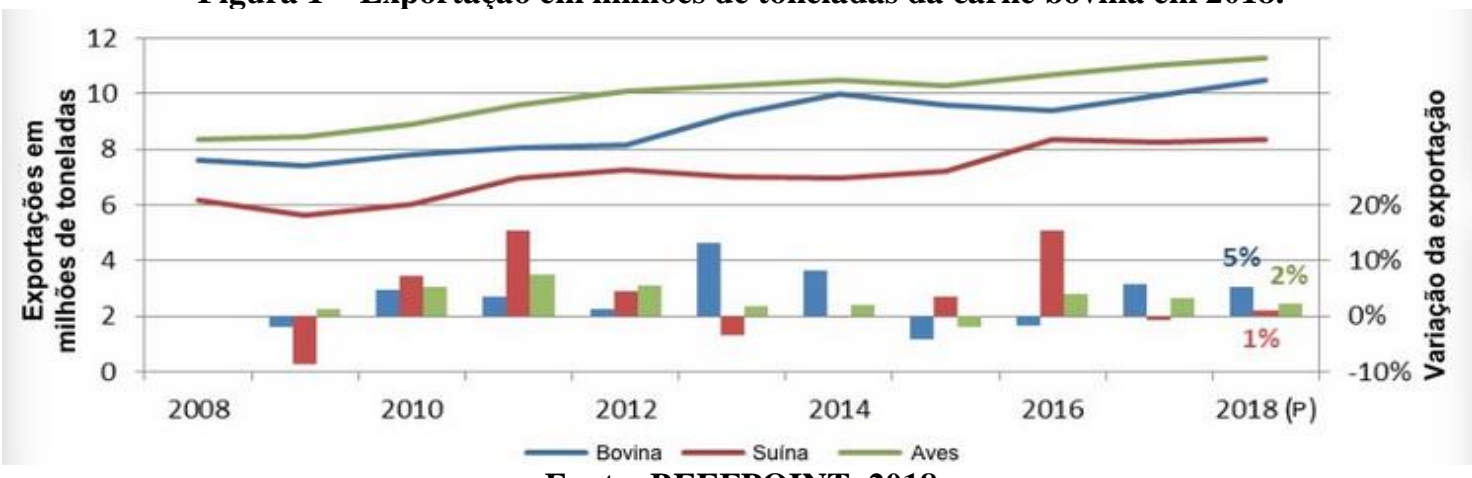

Fonte: BEEFPOINT, 2018

O cenário da agropecuária brasileira é gigante, repleto de oportunidade de investimento e desenvolvimento, possui uma expressiva participação na economia do país e representa cerca de 22,15\% do PIB (dados de 2012). Atualmente, o Brasil ocupa notável posição na produção agroindustrial mundial (Figura 2).

Figura 2 - Ranking do Brasil em produção agroindustrial.

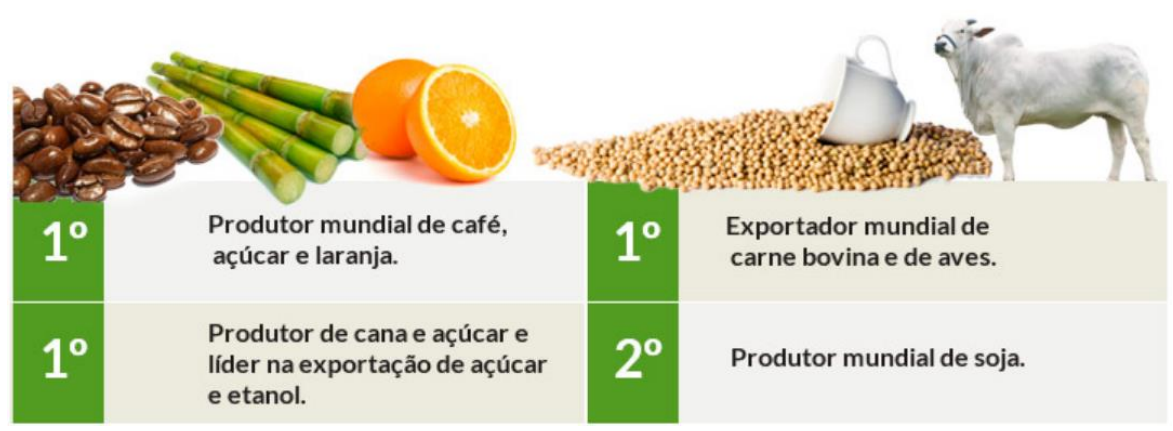

Fonte: ECOAGRO, 2018. 


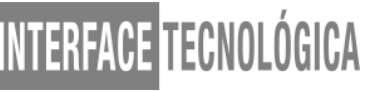

Além disso, o agronegócio no Brasil responde por cerca de 39\% das exportações, gerando em torno de $37 \%$ dos empregos do país, e no território nacional, com média de $30 \%$ das terras utilizadas para agropecuária (ECOAGRO, 2018).

\section{PROCEDIMENTOS METODOLÓGICOS}

\subsection{Apresentação do projeto}

Por meio da implantação do aplicativo, buscou-se entender a funcionalidade e atuação no segmento de rastreabilidade de bovinos.

A empresa irá atuar na propriedade, acompanhando o gado desde o seu nascimento, desenvolvimento, alimentação, crescimento, bem-estar, até a fase de conclusão do seu ciclo, sendo liberado para comercialização.

Esse gado terá um número de acompanhamento individual e por rebanho, onde o produtor poderá acompanhar todas as informações daquele animal/rebanho. Posteriormente, será gerado um relatório do lote comprado, com as principais informações a serem fornecidas.

O diferencial do aplicativo se faz por meio da prestação de serviço presencial e à distância, que será oferecido aos usuários.

\section{RESULTADOS E DISCUSSÃO}

\subsection{Utilização do aplicativo}

O aplicativo estará disponível nas plataformas Google Play e Apple Store, gratuito para download, porém, para a utilização de $100 \%$ dos serviços oferecidos, é necessário o pagamento de uma mensalidade, sendo essa, de acordo com o tamanho do rebanho, da propriedade e telas de acesso.

O mesmo foi desenvolvido exclusivamente para uso da empresa Agro Sky, possuindo uma conexão com os Drones e com os microchips implantados, disponibilizando todas as informações necessárias no seu smartphone, tablet ou notebook (Figura 3 e 4). 
Figura 3 - Layout do aplicativo desenvolvido.

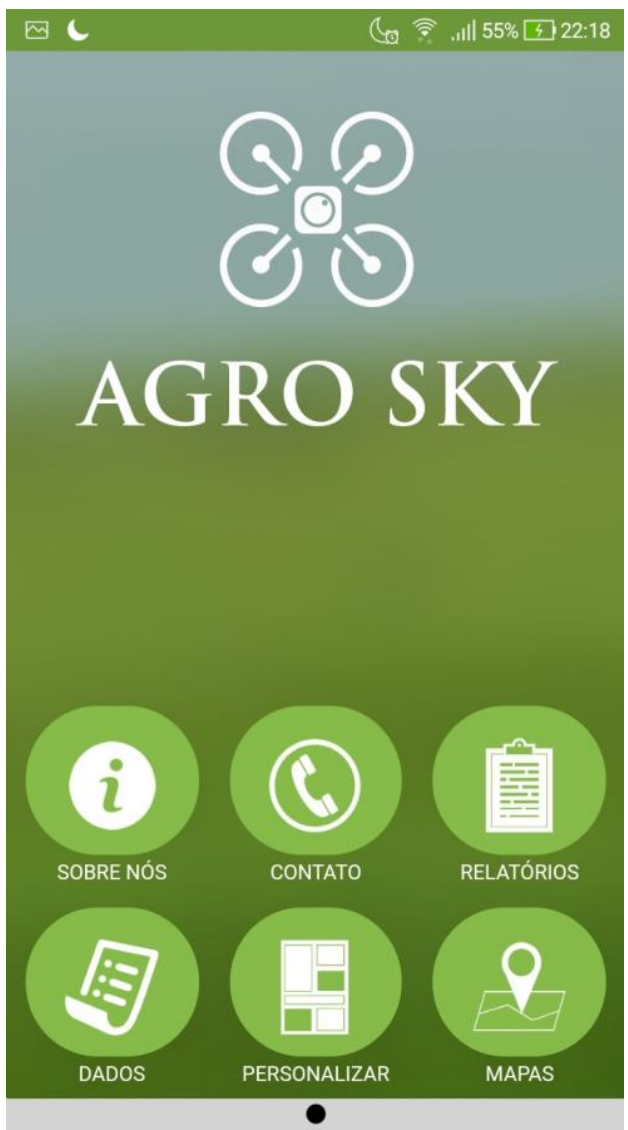

Fonte: Elaborado pelos autores, 2018.

Figura 4 - Fluxograma das atividades desenvolvidas pelo aplicativo.

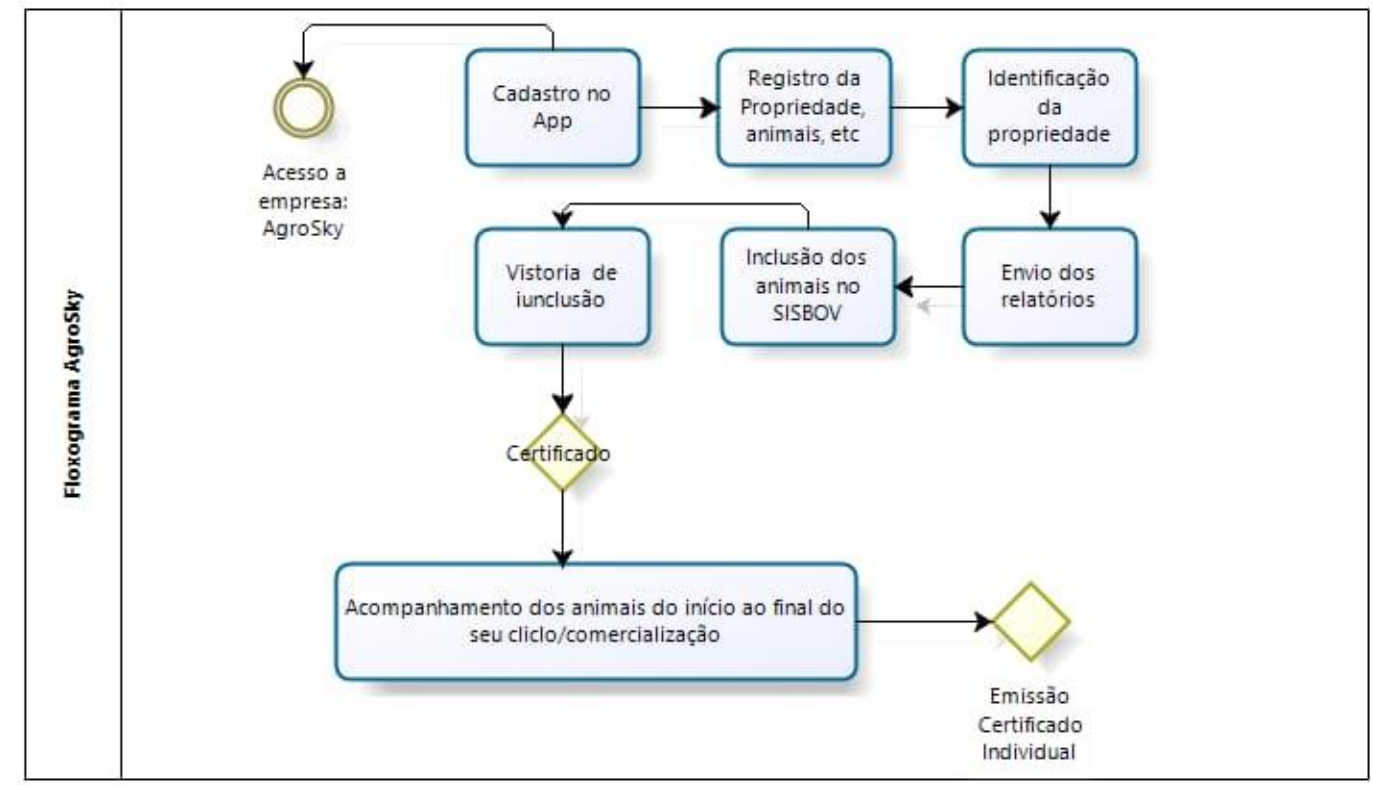

Fonte: Desenvolvido através do Bizagi pelos próprios autores, 2018. 


\section{WTEERFAET TECNOLLGGCA}

\subsection{Identificação do animal}

O SISBOV - Sistema Brasileiro de Identificação e Certificação de Origem Bovina e Bubalina, foi definido como um conjunto de ações, medidas e procedimentos necessários para definir a origem, o estado sanitário, a produtividade e a produção da pecuária nacional, garantindo a segurança dos alimentos. Essas medidas foram adotadas para melhorar o controle de origem e destino dos animais do produtor ao consumidor final, sendo possível o acesso via aplicativo (MOTA, 2011).

Em $1^{\circ}$ de Julho de 2004, foi definido que todos os animais devem ter uma identificação dupla. Nesse projeto, as identificações definidas foram um brinco auricular na orelha direita, devendo atender a padrões específicos das normas SISBOV, além de um dispositivo eletrônico, que devem obedecer às normas do sistema de qualidade, excelência técnica e normas específicas de padrão ISO (Figura 5), além de serem menos prejudiciais a carcaça do animal, se comparado com outras maneiras de identificação.

Figura 5 - Modelo do identificador individual de bovinos no SISBOV.

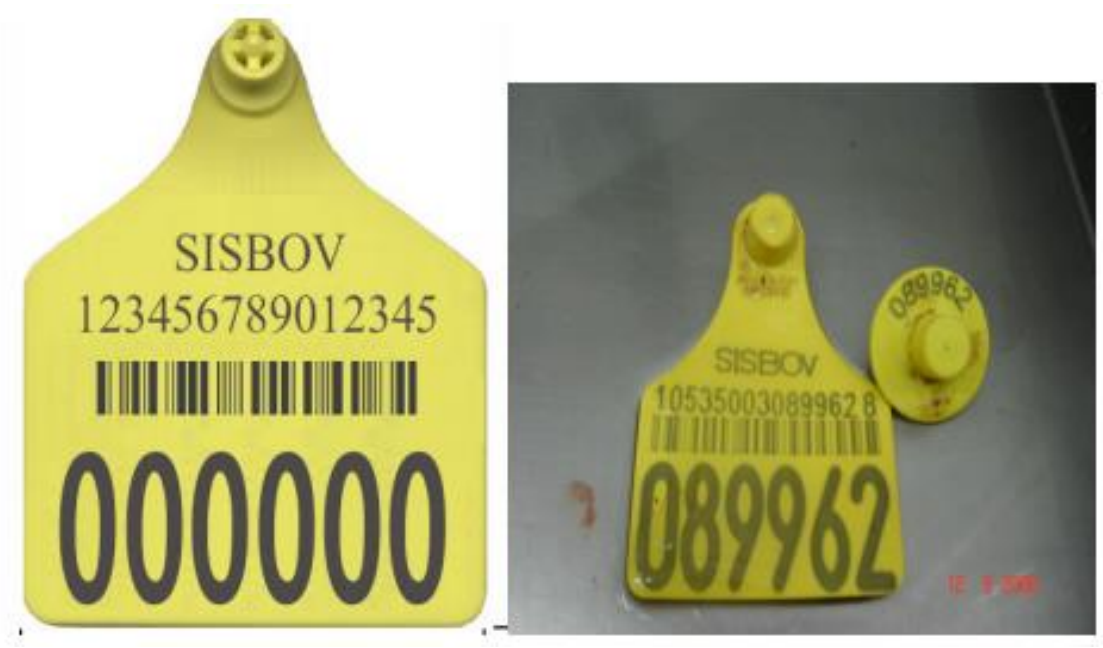

Fonte: MOTA, 2010.

\subsection{Drones}

A utilização da pecuária de precisão vem sendo cada vez mais frequente para auxiliar no gerenciamento de rebanhos, na busca de alimentos mais saudáveis, no controle adequado do negócio. Além disso, visa aumentar a produtividade e atendimento das demandas para consequente conquista de novos mercados, sejam eles internos ou externos (ABADE, 2015). 


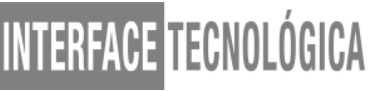

Sendo assim, é necessário a utilização de veículos não tripulados (VANT's ou Drones), para facilitar o monitoramento sobre o rebanho, que permitirá aos produtores acessarem o nosso sistema de gestão e informação, cujas informações foram captadas durante o tempo de vida do animal, apurando todos os dados, e assim podendo repassar quando necessário, garantindo qualidade e diferencial.

\section{CONCLUSÃO}

O aplicativo para a rastreabilidade tem uma interface de simples compreensão, fácil acesso e manuseio. A plataforma auxilia o produtor no controle do rebanho, através de relatórios diários do desenvolvimento e mudanças dos animais e locais.

Além de incluir o uso de tecnologia no dia a dia do produtor, agilizar os trabalhos a serem realizados no confinamento, reduzir custos e emitir um relatório do lote para as indústrias de abate, o projeto tem como finalidade facilitar a tomada de decisões da propriedade.

Com a implantação do aplicativo na propriedade agrícola, inicialmente o produtor passará a economizar tempo e irá possuir um maior controle sobre seu rebanho, além de existir a possibilidade de acessar todas as informações de seu lote mesmo não estando próximo ao gado. Além disto, a longo prazo, a implantação de tecnologia fará com que a possibilidade de erros seja reduzida a mínima, fazendo com que os custos na propriedade diminuam notoriamente.

\section{REFERÊNCIAS}

ABADE, A. S. Pecuária de precisão: o uso drones para o monitoramento de bovinos por meio de identificação eletrônica por rádio frequência. IV Feira IFMT de Inovação Tecnológica. Ed. 06. 2015.

BARCELLOS, J. O. J.; SUÑE, Y.B.P; SEMMELMANN C.E.N.A. Bovinocultura de corte frente a agriculturização no Sul do Brasil. In: XI CICLO DE ATUALIZAÇÃO EM MEDICINA VETERINÁRIA. 2, 2004, Lages, Anais... Lages: CAMEV - Centro Agroveterinário de Lages. 2004. 27p.

BEEFPOINT. USDA: Confira relatório sobre o mercado de carnes. Disponível em $<$ https://www.beefpoint.com.br/usda-confira-relatorio-sobre-o-mercado-de-carnes-2/> Acesso em 24 nov. 2018. 


\section{NTEBRFAEETECHOLOGGCA}

ECOAGRO. O Agronegócio no Brasil. 2018. Disponível em

<http://www.ecoagro.agr.br/agronegocio-brasil/>. Acesso em: 12 jun. 2018.

JUSTINO, D. Nas pegadas do boi. Safra - Revista do Agronegócio, Goiânia, n. 29, p. 32-34, abr. 2002.

LUCHIARI FILHO, A. Produção de carne bovina no Brasil, qualidade, quantidade ou ambas? In: SIMBOI. SIMPÓSIO SOBRE DESAFIOS E NOVAS TECNOLOGIAS NA BOVINOCULTURA DE CORTE, 2, 2006, Brasília, Anais... Brasília: SIMBOI, 2006.

MARTINS, F. M.; LOPES, M. A. Rastreabilidade bovina no Brasil. Boletim técnico. ed. 55. 2001.

MOTA, E.G.da. A rastreabilidade bovina no Brasil: histórico, evolução e perspectiva de futuro. 2011. 147 f., Dissertação (Mestrado em Agronegócios) - Universidade de Brasília, Brasília, 2011.

SERVIÇO BRASILEIRO DE CERTIFICAÇÕES. A importância da rastreabilidade bovina. 2018. Disponível em <http://sbcert.com.br/artigo-2/>. Acesso em: 25 nov. 2018. 